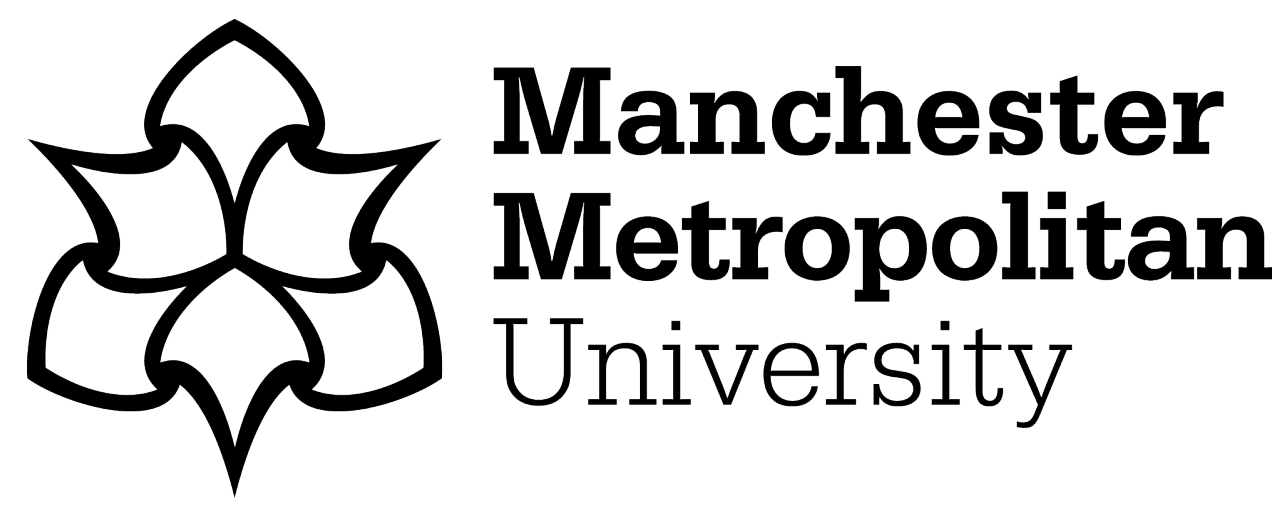

Dos'Santos, Thomas ORCID logoORCID: https://orcid.org/0000-0003-27150116, Thomas, Christopher and Jones, Paul A (2021) Assessing Interlimb Asymmetries: Are We Heading in the Right Direction? Strength and Conditioning Journal, 43 (3). pp. 91-100. ISSN 1524-1602

Downloaded from: https://e-space.mmu.ac.uk/626530/

Version: Accepted Version

Publisher: Lippincott, Williams \& Wilkins

DOI: https://doi.org/10.1519/SSC.0000000000000590

Usage rights: Creative Commons: Attribution-Noncommercial 4.0

Please cite the published version 


\title{
ASSESSING INTER-LIMB ASYMMETRIES: ARE WE HEADING IN THE RIGHT DIRECTION?
}

\begin{abstract}
The investigation of inter-limb asymmetries has increased in recent years, with practitioners and researchers typically exploring the impact of inter-limb asymmetry on athletic performance, screening inter-limb asymmetry in relation to injury risk, and monitoring of interlimb asymmetry during injury rehabilitation. A contentious issue regarding the profiling of inter-limb asymmetry is the use of thresholds to determine whether an athlete displays a 'meaningful' or potentially 'problematic' asymmetry and how to interpret such data. This article aims to outline the potential options available for practitioners and researchers regarding the quantification, monitoring, and interpretation of inter-limb asymmetries.
\end{abstract}

Keywords: between-limb difference; side-to-side difference; imbalance; symmetry; muscle strength asymmetry

\section{INTRODUCTION}

An inter-limb asymmetry is a difference in performance or function of one limb with respect to the other (17), and can be generally, but not exclusively, categorised into the following: anatomical or morphological asymmetries (e.g., lean mass difference between left and right limbs) $(38,39)$; flexibility asymmetries (e.g., ankle dorsi-flexion range of motion difference between left and right limbs) (40, 62); strength asymmetries (force asymmetries - e.g., isokinetic peak torque or countermovement jump [CMJ] propulsive force difference between left and right limbs); strategy asymmetries, (e.g., CMJ negative displacement or propulsion duration difference between left and right limbs); and skill or outcome asymmetries (e.g., difference in change of direction time or CMJ height between left and right limbs) $(40,55)$. Due to laterality, humans will preferentially use one side of the body when performing a motor 
task; typically resulting in more skilful and therefore dominant side $(26,38,55)$, thus it is unsurprising that athletes tend to display inter-limb asymmetries. In specific sporting contexts, inter-limb asymmetries arguably are a functional adaptation $(55,60)$, potentially due to the chronic exposure to repeated asymmetrical sport-specific actions, such as fencing (43) or badminton lunge and striking actions $(1,55)$, kicking actions in soccer $(4,5,25,58)$ and Australian rules football $(38,39)$, and asymmetrical throwing, bowling, and batting actions performed in cricket $(14,48)$.

Investigations into inter-limb asymmetry have increased in popularity over recent years, with research in this area typically focusing on the following: 1) impact of inter-limb asymmetry on athletic performance $(20,55) ; 2)$ the screening and profiling of inter-limb asymmetry in relation to injury risk $(23,40,41,47,49)$; and 3$)$ monitoring of limb function and inter-limb asymmetry during the injury rehabilitation process $(40,46,49,64,65)$; particularly rehabilitating from anterior cruciate ligament (ACL) injury (40, 46, 49, 65). Whether inter-limb asymmetries manifest into performance decrements and increases injury risk is a contentious issue, with mixed findings observed $(8,20,23,33,40,47,55)$, but nevertheless an asymmetry and imbalance can be viewed as a 'window for development' to improve the performance and function of the non-dominant limb (55). A plethora of work from researchers have outlined recommendations as to how to calculate, assess, and monitor interlimb asymmetries which has contributed to our insight and knowledge in this area $(6,8,11$, $16-22,55)$. However, we feel that there are gaps in the literature pertaining to the methods of quantifying, interpreting, and monitoring inter-limb asymmetry, and some of the associated issues when collecting and interpreting inter-limb asymmetry data need to be further highlighted. Additionally, and importantly, to the best of our knowledge, a framework for practitioners to help inform and assist in the preparation, assessment, analysis, and interpretation of inter-limb asymmetry data does not exist. 
The purpose of this article is to outline the potential options available for practitioners and researchers regarding the quantification, monitoring and interpretation of inter-limb asymmetries. Additionally, a further purpose is to outline a framework for practitioners to help inform and assist in the preparation, assessment, analysis, and interpretation of inter-limb asymmetry. Strength $(30,31,67)$, strategy $(16,56,65)$, and skill $(32,34,56,68)$ inter-limb asymmetries have been commonly investigated over recent years in the area of strength and conditioning and thus, will be the primary focus for this article.

\section{Is my athlete asymmetrical? Using asymmetry thresholds and asymmetry considerations}

A plethora of assessments are available to practitioners to evaluate strength and skill asymmetries in athletes $(19,40,44,67)$, and a range of equations are available for practitioners to evaluate inter-limb asymmetry which can result in different percentage magnitudes $(6,17$, $18,40)$. As such, practitioners are encouraged to follow the recommendations of previous work for calculating inter-limb asymmetries during unilateral and bilateral assessments $(18,40)$. When calculating an inter-limb asymmetry, a percentage figure is created, but a problematic issue for the practitioners is how to interpret this value (8). Traditionally, a $10-15 \%$ imbalance has been viewed as a potentially problematic asymmetry $(7,8,40,45,47,61)$, and this arbitrary threshold has been typically used to classify asymmetry over the last 20 years $(8,40)$. Recent work (8) has excellently highlighted the problems associated with using an arbitrary inter-limb threshold (i.e., 10-15\% threshold) which cannot be applied across all assessments and metrics, due to the problems of the task- and metric-dependent nature of inter-limb asymmetries $(2,16$, $30,31,35,37,40,67)$. As such, recent work regarding asymmetry thresholds has (8) advocated the approach recommended by Exell et al. (36) that an 'asymmetry might only be meaningful if the percentage value is greater than the test variability score' (i.e., coefficient of variation $[\mathrm{CV} \%])$, and recommends that an individual approach should be adopted when monitoring inter-limb asymmetries. 
Indeed, it is not disputed that that an arbitrary threshold cannot be universally applied across all assessments and metrics due to the task- and metric-dependent nature of inter-limb asymmetry $(16,40,67)$, and we agree that the inter-limb asymmetry percentage value should exceed the variability for the specific metric of a task. However, if an individual displays variability of $2 \%$ for a specific metric, with an inter-limb percentage difference of $3 \%$, although 'real', it in all probability does not represent at problematic asymmetry in terms of athletic performance and injury risk. In this example, an asymmetry value so low and close to zero would be questionable to suggest using solely such an approach when interpreting an individual's inter-limb asymmetry. This is pertinent when research examining whether asymmetry is a problem does not reveal a clear relationship, particularly because typical asymmetries for certain metrics and tasks are relatively low $(20,31,35,40,55,67)$. Alternative methods we feel are applicable, or methods that have been used previously, will be discussed in the following sections to assist in the interpretation of inter-limb asymmetry.

While we agree that an individual approach is needed regarding the monitoring of interlimb asymmetries $(8,11)$, and monitoring of variables in general in strength and conditioning (i.e., training load, fatigue, interventions etc.) (57), it is difficult to adopt such an approach and interpret data without benchmark, criteria, or normative data related to the metric, task, and population in question. Unfortunately, recent work which has discussed the concept of interlimb asymmetry thresholds (8) fails to discuss some asymmetry thresholds that have been utilised in the literature, including thresholds for metrics based on the population mean + smallest worthwhile change $(\mathrm{SWC})(0.2 \times$ between-subject standard deviation $[\mathrm{SD}])(31,35$, $40,51,52)$, mean $+(0.5 \times$ between-subject SD) $(40)$, or population mean $+(1.0 \times$ betweensubject $\mathrm{SD})(2,37,40,67,68)$. Assuming the associated asymmetry of the metric is normally distributed, the proportion of athletes deemed 'asymmetrical' based on the abovementioned thresholds would be $\sim 42 \%, \sim 31 \%$, and $\sim 16 \%$, respectively, and may provide practitioners with 
the option to classify individuals in their population with 'small to moderate' or 'high or extreme' asymmetries. The aforementioned thresholds have been used to establish benchmarks in athletic populations, and have been used to assess the agreement in the direction of asymmetry between tasks and metrics $(2,31,35,37,67)$; highlighting the task- and metricdependent nature of inter-limb asymmetry.

It is important to note that inter-limb asymmetry may not always be parametric because of the range of asymmetry values observed for particular metrics and athletic populations and the small sample sizes often used by researchers and practitioners. Thus, practitioners should ensure that they inspect the normality of their inter-limb asymmetry data when adopting and aiming to use the aforementioned thresholds. Practitioners with non-parametric data may consider looking at the median and interquartile range of their data to assist in the interpretation of inter-limb asymmetries for a specific metric within their population. In this scenario, the upper quartile could be used to represent athletes with potentially 'high or extreme' asymmetries.

We are not, by any means, suggesting that this a perfect solution, but the approach of using the mean + SWC and mean + SD for asymmetry thresholds allows practitioners to create benchmarks, criteria, and normative data that are specific to their athlete population for a range of metrics and tests. Specially, the aforementioned thresholds allow practitioners a method of classifying athletes with 'small to moderate' and 'extreme or high' asymmetries, respectively. The mean + SWC threshold is a more sensitive approach which will classify a greater proportion of athletes $(\sim 42 \%)$ with 'small to moderate' inter-limb asymmetries. Conversely, the mean $+(1.0 \times$ between-subject SD) threshold is a more conservative approach which will classify a smaller proportion $(\sim 16 \%)$ of athletes with 'high or extreme' inter-limb asymmetries. It should be noted that neither threshold is superior, but both should be used in combination to provides descriptors to the magnitude of the asymmetry (for example, similar to the thresholds 
and descriptors used for an effect size scale) to assist in the interpretation and classification of inter-limb asymmetry. The concept of interpreting data relative to the population mean and using the SWC for monitoring changes in fitness and training load data is common practice in Sports Science and Strength and Conditioning (57). Consequently, athletes that display interlimb asymmetries greater than the population mean + SWC, and their individual variability $(\mathrm{CV} \%)$, can therefore be potentially interpreted as displaying 'meaningful' or 'greater' asymmetry in context of the population and metric for a specific test.

While investigating inter-limb asymmetry is indeed insightful into athletes' physical profiling and has been advocated in sporting and clinical setting $(19,22,40)$, it is important that practitioners should are aware of some key considerations that may influence the evaluation of inter-limb asymmetry profiles (Figure 1). For example, previous work has highlighted that the magnitudes and directions of inter-limb asymmetry are not necessarily consistent between-sessions (16), and are also sensitive to acute (12) and post-match fatigue (immediate, 24, and 48 hours post fixture) (24). As such, practitioners are encouraged to establish the between-session reliability for inter-limb asymmetries to ensure that the magnitude (i.e., \% imbalance) and direction (limb displaying greater function or performance) is consistent between sessions before classifying athletes as asymmetrical. It is recommended that a combination of statistical tests are used to assess for systematic bias (e.g., paired sample t-test or non-parametric equivalent), within-subject variation (e.g., CV\%, typical error, or standard error of measurement), and retest correlation (e.g., intraclass correlation coefficient) for a more holistic overview of reliability $(3,42,53)$. Additionally, practitioners should ensure that they evaluate their athletes' inter-limb asymmetries in a non-fatigued state $(12,24)$; ensuring that their athletes are sufficiently recovered from competitive matches so that any large imbalances observed are not attributed to fatigue. Finally, practitioners should acknowledge that asymmetries may fluctuate throughout a competitive season or macrocycle 
(15), though further research is necessary to better understand how asymmetry changes throughout the season and macrocycle.

$* * *$ Insert Figure 1 here***

\section{Inter-limb asymmetry: data visualisation and interpretation}

Recent work (8) has presented illustrative methods for interpreting individuals' asymmetry values relative to their variability. We agree that this is a useful method for illustrating and monitoring inter-limb asymmetries; however, we feel that the addition of asymmetry threshold lines (mean + SWC) and 'high' asymmetry threshold lines (mean + SD) are useful additions to assist in the interpretation of asymmetries for athletes in a specific population (40). For example, Figure 2 illustrates hypothetical individual unilateral isometric mid-thigh pull (IMTP) peak force (PF) inter-limb asymmetry values, individual CV\% values, and the asymmetry thresholds calculated on the population mean + SWC and mean + SD. The mean asymmetry IMTP PF value was $6.5 \pm 3.9 \%$, resulting in asymmetry thresholds of $7.3 \%$ (small to moderate) and $10.4 \%$ (high / extreme) which is specific to this population and metric during this test. In this example, 11 athletes displayed asymmetries greater than their individual CV\%, but only 6 athletes displayed asymmetries which exceeded the asymmetry threshold (Figure 2). However, it is worth noting that, despite displaying an asymmetry greater than the asymmetry threshold, the asymmetry for Athlete 6 was not greater than the $\mathrm{CV} \%$, and therefore cannot be interpreted as 'real'. As such, 2 athletes (Athlete 8 and 11) displayed asymmetries greater than their individual CV\% and 'small to moderate' asymmetry threshold, while 3 athletes (Athlete 1, 7, 12) displayed 'high' asymmetries which were also greater than their individual CV\% (Figure 2). However, practitioners should be mindful that if the $\mathrm{CV} \%$ is $>10 \%$, therefore considered unacceptable absolute reliability $(29,59)$, interpreting asymmetry for that metric and / or athlete is likely sub-optimal. Therefore, it is advised that practitioners investigate metrics during tasks 
that display low variability, and ensure that athletes are adequately familiarized with the task and assessment procedures to improve data collection and subsequent analysis and interpretation.

***Insert Figure 2 here***

Alternatively, practitioners may also consider calculating Z-scores of their inter-limb asymmetries for their population, to identify which athletes display asymmetries greater than average (Figure 3), and may consider using Z-score benchmarks of $0.20-0.99$, and $\geq 1.00$ to classify athletes with 'small to moderate' and 'extreme or high' asymmetries (40), respectively. The procedure of using Z-scores is commonly advocated and used in practice when interpreting strength, power, and fitness data $(57,69)$.

*** Insert Figure 3 here***

\section{Inter-limb asymmetry is a percentage / ratio: do not forget the absolute components when interpreting and profiling}

While the inter-limb percentage figure is indeed a simple method for indicating deficiencies in performance between the dominant and the non-dominant limb, and a useful communication approach, the above-mentioned proposed asymmetry thresholds calculations can also be applied to raw scores. Statistically, a raw score may be more accurate to consider as this will not be dependent on changes to the denominator; therefore, arguably, a percentage index may only be appropriate for monitoring changes in inter-limb asymmetry if the dominant limb increases or decreases (either between or within subjects) in direct proportion to the nondominant limb for a metric of interest. Practitioners are encouraged to refer to previous work which acknowledges some of the issues of calculating percentage differences and percentage changes $(27,28)$. As such, it is recommended that the absolute components (i.e., numerator 
and denominator) must also be acknowledged and considered when interpreting and making decisions regarding an athlete's physical profile (Figure 1).

As previously stated, when interpreting an athlete' physical profile, it is critical that coaches interpret the absolute components (i.e., numerator and denominator). For example, an athlete may be strong or display high function or performance and asymmetrical, but their weaker or lower functioning limb may still outperform the rest of the squad or exceeds normative data or benchmark criteria. Nevertheless, the non-dominant limb can still be viewed as a 'window for development' (55). Conversely, an athlete may display similar strength and function or performance between limbs and would therefore be classed symmetrical, but inspection of the absolute measures may indicate that the athlete is weak or has poor function and the worst performer for that metric in the squad or when compared to normative data. Arguably, the symmetrical but weak or poor function athlete arguably warrants greater attention and strength and conditioning support. Figure 4 illustrates four quadrants to assist in the physical profiling of athletes. The optimal scenario for practitioners is athletes who are equally strong and displaying high function and performance from both limbs, which should be viewed as the ultimate aim for strength and conditioning practitioners (Figure 4 - green). Conversely, the worst case and 'red flag' scenario for practitioners is identifying a weak or low function and asymmetrical athlete (Figure 4 - red).

***Insert Figure 4 here***

It should be noted that although an athlete's magnitude of asymmetry may not change longitudinally, the absolute components may increase which can still be viewed as positive adaptation because the athlete has improved their strength, function, or performance in both limbs proportionately. Equally, however, athletes may maintain similar asymmetry values longitudinally, but the absolute components have reduced proportionately (i.e., weaker, 
reduced function, or performance in both limbs) which is problematic with respect to athletic performance and potential injury risk $(54,66)$ (Figure 5a). Additionally, from an injury rehabilitation monitoring perspective, athletes have been reported to display reductions in interlimb asymmetries (limb-symmetry index) during rehabilitation where this was attributed to reductions (detraining) in strength and performance of the contralateral (i.e., dominant limb) limb (64). This is therefore problematic, particularly in the context of ACL injury, as the contralateral limb is typically at greater risk of injury following an ACL injury (63). Thus, practitioners should ensure that when monitoring inter-limb asymmetries, they inspect the absolute (raw) values because an athlete can reduce their \% imbalance via reductions in dominant limb strength, performance, or function, while maintaining strength, performance, or function in the non-dominant limb (64) (Figure 5b).

***Insert Figure 5 here***

\section{Direction of asymmetry: future directions for research}

Finally, we agree that the direction of asymmetry is a key factor to acknowledge when monitoring inter-limb asymmetries (i.e., asymmetry favouring the same limb between metrics and / or tasks) $(8,22)$ and recent work $(8)$ advocates the use of Kappa coefficients to assess the agreements in limb dominance (i.e., limb producing greatest value or best performance) between metrics, tasks, and sessions to assist in inter-limb asymmetry profiling. However, previous research which has utilised this approach have only determined if the direction of asymmetry favours the left or right limb between tasks, metrics, trials, or sessions, but not quantified the agreements in 'real' asymmetries (i.e., did the same limb exceed the CV\% for both metrics / task / sessions) $(9-11,13,16,50)$. Thus, it appears that agreements in limb dominance performed by previous studies have classified limbs as left or right dominant without accounting for individual variability $(9-11,13,16)$. Consequently, as emphasis has 
been placed on ensuring the inter-limb asymmetry exceeds the variability $(8,22,36)$, it would be more suitable to assess agreements in 'real' asymmetries for left and right limbs between metrics, tasks, or sessions, with inter-limb asymmetries $\leq \mathrm{CV} \%$ considered 'trivial', 'unclear', or 'balanced'. Additionally, it may also be worthwhile to assess the agreements in asymmetries greater than the asymmetry thresholds (i.e., mean $+\mathrm{SWC}$ and / or mean $+\mathrm{SD}$ ) to improve our understanding regarding the direction of inter-limb asymmetries.

\section{PRACTICAL APPLICATIONS}

Whether inter-limb asymmetries manifest into performance decrements and increases injury risk is a contentious issue $(8,20,23,33,40,47,55)$, but for most athletes it would advantageous to be equally strong and possess high function and performance for both left and right limbs. As such, an asymmetry can be viewed as a 'window for development' and may warrant greater training focus to improve the performance and function of the non-dominant limb (Figure 4) (55). However, it should be noted that the dominant limb should not be neglected, and practitioners should still seek to improve performance and function in the dominant limb.

The investigation of inter-limb asymmetry has increased in popularity over recent years, with a plethora of articles outlining practical recommendations for the assessment, calculation, and interpretation of inter-limb asymmetries $(6,8,11,16-22,55)$. The primary purpose of this article was to outline the potential options available for practitioners and researchers regarding the quantification, monitoring and interpretation of inter-limb asymmetries. In summary, Figure 1 presents key considerations related to the task, athlete, and interpretation which should be acknowledged by practitioners when monitoring inter-limb asymmetries. Figure 6 presents a framework for practitioners to help inform and assist in the preparation, assessment, analysis, and interpretation of inter-limb asymmetry data. 
Consequently, to improve inter-limb asymmetry profiling and research, we suggest that practitioners and researchers should consider the following recommendations:

1. Create asymmetry thresholds based on population mean + SWC $(0.2 \times$ between-subject $\mathrm{SD})$ and population mean $+(1.0 \times$ between-subject SD) to allows practitioners a method of classifying athletes with 'small to moderate' and 'extreme or high' asymmetries. This enables practitioners to create benchmarks, criteria, and normative data that are specific to their athlete population for a range of metrics and tests. The mean $+\mathrm{SWC}$ threshold is a more sensitive approach which will classify a greater proportion $(\sim 42 \%)$ of athletes with 'small to moderate' inter-limb asymmetries. Conversely, the mean $+(1.0 \times$ between-subject SD) threshold is a more conservative approach which will classify a smaller proportion $(\sim 16 \%)$ of athletes with 'high or extreme' inter-limb asymmetries. It should be noted that neither threshold is superior, but both should be used in combination to provides descriptors to the magnitude of the asymmetry (similar to the thresholds and descriptors used for an effect size scale) to assist in the interpretation and classify of inter-limb asymmetry.

2. Athletes which display asymmetries which exceed their individual $\mathrm{CV} \%$ and population asymmetry (mean + SWC / mean $+\mathrm{SD}$ ) can be interpreted as displaying 'small to moderate' or 'high or extreme' asymmetries.

3. Practitioners may consider creating combined column and line charts (Figure 2) as a datavisualisation method for interpreting inter-limb asymmetry. Additionally, practitioners should also consider using Z-scores when interpreting inter-limb asymmetry (Figure 3).

4. Practitioners should consider establishing the between-session reliability for inter-limb asymmetry metrics to ensure that the magnitude and direction of asymmetry is consistent between sessions. Consequently, it is recommended that a combination of statistical tests (e.g. statistical tests for systematic bias, within-subject variation, and retest correlation) are used for a more holistic overview of reliability $(3,42,53)$. Additionally, when collecting 
inter-limb asymmetry data, practitioners should ensure that data is collected in a nonfatigued state, with data collected with sufficient recovery from previous matches (i.e., $>96$ hours).

5. Practitioners are advised to evaluate the absolute components (raw values) of the inter-limb asymmetry metric, in addition to the percentage value, to assist in physical profiling and training prescription (Figures $4 \& 5$ ).

6. Acknowledge the key factors which can impact the collection and interpretation of interlimb asymmetry data (Figure 1), and use the framework (Figure 6) to help inform and assist in the preparation, assessment, analysis, and interpretation of inter-limb asymmetry data.

Conflicts of Interest and Source of Funding: The authors report no conflicts of interest and no source of funding.

\section{REFERENCES}

1. Abián P, Abián-Vicén J, and Sampedro J. Anthropometric analysis of body symmetry in badminton players. Int J Morphol 30: 945-951, 2012.

2. Aldukhail A, Jones P, Gillard H, and Graham-Smith P. Clinical diagnosis of strength and power asymmetry. Biol Sport 15: 33-38, 2013.

3. Atkinson $\mathrm{G}$ and Nevill AM. Statistical methods for assessing measurement error (reliability) in variables relevant to sports medicine. Sports Med 26: 217-238, 1998.

4. Ball K. Loading and performance of the support leg in kicking. J Sci Med Sport 16: 455-459, 2013.

5. Ball KA. Kinematic comparison of the preferred and non-preferred foot punt kick. J Sports Sci 29: 1545-1552, 2011.

6. Bampouras TM and Dewhurst S. A comparison of bilateral muscular imbalance ratio calculations using functional tests. J Strength Cond Res 32: 2216-2220, 2018.

7. Barber S, Noyes FR, Mangine RE, McCloskey J, and Hartman W. Quantitative assessment of functional limitations in normal and anterior cruciate ligament-deficient knees. Clin Orthop Relat: 204-214, 1990.

8. Bishop C. Interlimb Asymmetries: Are Thresholds a Usable Concept? Strength Cond J: Epub Ahead of Print, 2020.

9. Bishop C, Abbott W, Brashill C, Turner AN, Lake J, and Read P. Bilateral vs. unilateral countermovement jumps: comparing the magnitude and direction of asymmetry in elite academy soccer players. J Strength Cond Res: Epub ahead of print, 2020.

10. Bishop C, Gullon I, Maloney S, and Balsalobre-Fernandez C. Jump and Change of Direction Speed Asymmetry Using Smartphone Apps: Between-Session Consistency and Associations With Physical Performance. J Strength Cond Res: Epub ahead of print, 2020.

11. Bishop C, Lake J, Loturco I, Papadopoulos K, Turner AN, and Read P. Interlimb asymmetries: The need for an individual approach to data analysis. J Strength Cond Res: Epub Ahead of Print, 2018. 
12. Bishop C, McCauley W, Read P, Gonzalo-Skok O, Lake JP, and Turner A. The acute effects of repeated sprints on inter-limb asymmetries during unilateral jumping. J Strength Cond Res: Epub ahead of print, 2019.

13. Bishop C, Pereira LA, Reis VP, Read P, Turner AN, and Loturco I. Comparing the magnitude and direction of asymmetry during the squat, countermovement and drop jump tests in elite youth female soccer players. J Sports Sci: Epub ahead of print, 2019.

14. Bishop C, Read P, Brazier J, Jarvis P, Chavda S, Bromley T, and Turner A. Effects of inter-limb asymmetries on acceleration and change of direction speed: a between-sport comparison of professional Soccer and Cricket athletes. J Strength Cond Res: Epub ahead of print, 2019.

15. Bishop C, Read P, Chavda S, Jarvis P, Brazier J, Bromley T, and Turner A. Magnitude or Direction? Seasonal Variation of Interlimb Asymmetry in Elite Academy Soccer Players. J Strength Cond Res: Epub ahead of print, 2020.

16. Bishop C, Read P, Chavda S, Jarvis P, and Turner A. Using unilateral strength, power and reactive strength tests to detect the magnitude and direction of asymmetry: $A$ test-retest design. Sports 7: 58, 2019.

17. Bishop C, Read P, Chavda S, and Turner A. Asymmetries of the Lower Limb: The Calculation Conundrum in Strength Training and Conditioning. Strength Cond J 38: 27-32, 2016.

18. Bishop C, Read P, Lake J, Chavda S, and Turner A. Inter-limb asymmetries: understanding how to calculate differences from bilateral and unilateral tests. Strength Cond J 40: 1-6, 2018.

19. Bishop C, Turner A, Jarvis P, Chavda S, and Read P. Considerations for selecting field-based strength and power fitness tests to measure asymmetries. J Strength Cond Res 31: 26352644, 2017.

20. Bishop C, Turner A, and Read P. Effects of inter-limb asymmetries on physical and sports performance: a systematic review. J Sports Sci 36: 1135-1144, 2017.

21. Bishop C, Turner A, and Read P. Training Methods and Considerations for Practitioners to Reduce Interlimb Asymmetries. Strength Cond J 40: 40-46, 2018.

22. Bishop C, Turner AN, Gonzalo-Skok $O$, and Read P. Inter-limb asymmetry during rehabilitation understanding formulas and monitoring the" Magnitude" and" Direction". Aspetar Sports Med J 9: 18-22, 2020.

23. Bourne MN, Opar DA, Williams MD, and Shield AJ. Eccentric knee flexor strength and risk of hamstring injuries in rugby union: a prospective study. Am J Sport Med 43: 2663-2670, 2015.

24. Bromley T, Turner A, Read P, Lake J, Maloney S, Chavda S, and Bishop C. Effects of a competitive soccer match on jump performance and interlimb asymmetries in elite academy soccer players. J Strength Cond Res: Epub ahead of print, 2019.

25. Brophy RH, Backus SI, Pansy BS, Lyman S, and Williams RJ. Lower extremity muscle activation and alignment during the soccer instep and side-foot kicks. J Orthop Sport Phys 37: 260-268, 2007.

26. Carpes FP, Mota CB, and Faria IE. On the bilateral asymmetry during running and cycling-A review considering leg preference. Phys Ther Sport 11: 136-142, 2010.

27. Cole TJ and Altman DG. Statistics notes: percentage differences, symmetry, and natural logarithms. BMJ 358: j3683, 2017.

28. Cole TJ and Altman DG. Statistics Notes: What is a percentage difference? BMJ 358: j3663, 2017.

29. Cormack SJ, Newton RU, McGuigan MR, and Doyle TL. Reliability of measures obtained during single and repeated countermovement jumps. Int J Sports Physiol and Perform 3: 131144, 2008.

30. Dos'Santos T, Thomas C, Jones PA, and Comfort P. Assessing muscle strength asymmetry via a unilateral stance isometric mid-thigh pull. Int J Sports Physiol and Perform 12: 505-511, 2017. 
31. Dos'Santos T, Thomas C, Jones PA, and Comfort P. Asymmetries in isometric force-time characteristics are not detrimental to change of direction speed. J Strength Cond Res 32: 520-527, 2018.

32. Dos'Santos T, Thomas C, Jones PA, and Comfort P. Assessing Asymmetries in Change of Direction Speed Performance; Application of Change of Direction Deficit. J Strength Cond Res 33: 2953-2961, 2019.

33. Dos'Santos T, Bishop C, Thomas C, Comfort P, and Jones PA. The effect of limb dominance on change of direction biomechanics: a systematic review of its importance for injury risk. Phys Ther Sport 37: 179-189, 2019.

34. Dos'Santos T, Thomas C, Comfort P, and Jones PA. Comparison of change of direction speed performance and asymmetries between team-sport athletes: application of change of direction deficit. Sports 6: 174, 2018.

35. Dos'Santos T, Thomas C, Jones PA, and Comfort P. Asymmetries in single and triple hop are not detrimental to change of direction speed. J Trainol 6: 35-41, 2017.

36. Exell TA, Irwin G, Gittoes MJ, and Kerwin DG. Implications of intra-limb variability on asymmetry analyses. J Sports Sci 30: 403-409, 2012.

37. Graham-Smith P, Al-Dukhail A, and Jones P. Agreement between attributes associated with bilateral jump asymmetry. Presented at ISBS-Conference Proceedings Archive, Poitiers, France, 29 June - 3 July 2015.

38. Hart NH, Newton RU, Weber J, Spiteri T, Rantalainen T, Dobbin M, Chivers P, and Nimphius S. Functional Basis of Asymmetrical Lower-Body Skeletal Morphology in Professional Australian Rules Footballers. J Strength Cond Res 34: 791-799, 2020.

39. Hart NH, Nimphius S, Spiteri T, and Newton RU. Leg strength and lean mass symmetry influences kicking performance in Australian football. J Sport Sci Med 13: 157-165, 2014.

40. Herrington LC, Munro AG, and Jones PA. Assessment of factors associated with injury risk, in: Performance Assessment in Strength and Conditioning. P Comfort, JJ McMahon, PA Jones, eds. Abingdon, Oxon, United Kingdom: Routledge, 2018, pp 53-95.

41. Hewett T, Myer GD, Ford KR, Heidt RS, Colosimo AJ, McLean SG, Van den Bogert AJ, Paterno $M V$, and Succop P. Biomechanical measures of neuromuscular control and valgus loading of the knee predict anterior cruciate ligament injury risk in female athletes a prospective study. Am J Sport Med 33: 492-501, 2005.

42. Hunter JP, Marshall RN, and McNair P. Reliability of biomechanical variables of sprint running. Med Sci Sports Exerc 36: 850-861, 2004.

43. Iglesias $X$, Irurtia A, Marina $M$, and Carrasco M. Bilateral deficit and morphofunctional asymmetries in young fencers. Apunts Medicina de I" Esport (English Edition) 46: 65-71, 2011.

44. Jones PA and Bampouras TM. A comparison of isokinetic and functional methods of assessing bilateral strength imbalance. J Strength Cond Res 24: 1553-1558, 2010.

45. Kannus P. Isokinetic evaluation of muscular performance: implications for muscle testing and rehabilitation. Int J Sports Med 15: S11-18, 1994.

46. King E, Richter C, Franklyn-Miller A, Wadey R, Moran R, and Strike S. Back to normal symmetry? Biomechanical variables remain more asymmetrical than normal during jump and change-of-direction testing 9 months after anterior cruciate ligament reconstruction. Am J Sport Med 47: 1175-1185, 2019.

47. Knapik JJ, Bauman $\mathrm{CL}$, Jones $\mathrm{BH}$, Harris JM, and Vaughan L. Preseason strength and flexibility imbalances associated with athletic injuries in female collegiate athletes. Am J Sport Med 19: 76-81, 1991.

48. Kountouris A, Portus M, and Cook J. Cricket fast bowlers without low back pain have larger quadratus lumborum asymmetry than injured bowlers. Clin J Sport Med 23: 300-304, 2013. 
49. Kyritsis P, Bahr R, Landreau P, Miladi R, and Witvrouw E. Likelihood of ACL graft rupture: not meeting six clinical discharge criteria before return to sport is associated with a four times greater risk of rupture. Br J Sports Med 50: 946-951, 2016.

50. Lake JP, Mundy PD, Comfort P, and Suchomel TJ. Do the peak and mean force methods of assessing vertical jump force asymmetry agree? Sport Biomech 19: 227-234, 2020.

51. Lockie RG, Callaghan SJ, Berry SP, Cooke ERA, Jordan CA, Luczo TM, and Jeffriess MD. Relationship Between Unilateral Jumping Ability and Asymmetry on Multidirectional Speed in Team-Sport Athletes. J Strength Cond Res 28: 3557-3566, 2014.

52. Lockie RG, Schultz AB, Callaghan SJ, and Jeffriess MD. The effects of isokinetic knee extensor and flexor strength on dynamic stability as measured by functional reaching. Isokinet Exerc Sci 21: 301-309, 2013.

53. Luiz RR and Szklo M. More than one statistical strategy to assess agreement of quantitative measurements may usefully be reported. J Clin Epidemiol Number 58: 215-216, 2005.

54. Malone S, Hughes B, Doran DA, Collins K, and Gabbett TJ. Can the workload-injury relationship be moderated by improved strength, speed and repeated-sprint qualities? I Sci Med Sport 22: 29-34, 2019.

55. Maloney SJ. The Relationship Between Asymmetry and Athletic Performance: A Critical Review. J Strength Cond Res 33: 2579-2593, 2019.

56. Maloney SJ, Richards J, Nixon DG, Harvey L, and Fletcher IM. Do stiffness and asymmetries predict change of direction performance? J Sports Sci 35: 547-556, 2016.

57. McGuigan M. Monitoring training and performance in athletes. Champaign, Illinois: Human Kinetics, 2017.

58. McLean B and Tumilty D. Left-right asymmetry in two types of soccer kick. Br J Sports Med 27: 260-262, 1993.

59. Mundy P and Clarke ND. Reliability, valdity and measurement error, in: Performance Assessment in Strength and Conditioning. P Comfort, PA Jones, JJ McMahon, eds. Abdingdon, Oxon, United Kingdom: Routledge, 2019, pp 23-32.

60. Newton RU, Gerber A, Nimphius S, Shim JK, Doan BK, Robertson M, Pearson DR, Craig BW, Häkkinen $\mathrm{K}$, and Kraemer WJ. Determination of functional strength imbalance of the lower extremities. J Strength Cond Res 20: 971-977, 2006.

61. Noyes FR, Barber SD, and Mangine RE. Abnormal lower limb symmetry determined by function hop tests after anterior cruciate ligament rupture. Am J Sport Med 19: 513-518, 1991.

62. Overmoyer GV and Reiser RF. Relationships between lower-extremity flexibility, asymmetries, and the Y balance test. J Strength Cond Res 29: 1240-1247, 2015.

63. Paterno MV, Rauh MJ, Schmitt LC, Ford KR, and Hewett TE. Incidence of second ACL injuries 2 years after primary ACL reconstruction and return to sport. Am J Sport Med 42: 1567-1573, 2014.

64. Patterson BE, Crossley KM, Perraton L, Kumar AS, King MG, Heerey JJ, Barton CJ, and Culvenor AG. Limb symmetry index on a functional test battery improves between one and five years after anterior cruciate ligament reconstruction, primarily due to worsening contralateral limb function. Phys Ther Sport 44: 67-74, 2020.

65. Read PJ, Michael Auliffe S, Wilson MG, and Graham-Smith P. Lower Limb Kinetic Asymmetries in Professional Soccer Players With and Without Anterior Cruciate Ligament Reconstruction: Nine Months Is Not Enough Time to Restore "Functional" Symmetry or Return to Performance. Am J Sport Med: 0363546520912218, 2020.

66. Suchomel TJ, Nimphius S, and Stone MH. The Importance of Muscular Strength in Athletic Performance. Sports Med 46: 1419-1449, 2016.

67. Thomas C, Comfort P, Dos'Santos T, and Jones PA. Determining bilateral strength imbalances in youth basketball athletes. Int J Sports Med 38: 683-690, 2017. 
68. Thomas C, Dos' Santos T, Comfort P, and Jones PA. Effect of Asymmetry on Biomechanical Characteristics During 180 Change of Direction. J Strength Cond Res 34: 1297-1306, 2020.

69. Turner AN, Jones B, Stewart P, Bishop C, Parmar N, Chavda S, and Read P. Total score of athleticism: holistic athlete profiling to enhance decision-making. Strength Cond J 41: 91101, 2019. 